\title{
ESTILOS ALTERNATIVOS DE DESARROLLO LOCAL: METODOLOGÍA UTILIZADA PARA EL CASO DE UNA INVESTIGACIÓN ACCIÓN PARTICIPATIVA EN LA PROVINCIA DE MÁLAGA
}

\author{
ALTERNATIVE STYLES OF LOCAL DEVELOPMENT: \\ METHODOLOGY USED FOR THE CASE OF A RESEARCH \\ PARTICIPATORY ACTION IN THE PROVINCE OF MÁLAGA
}

\author{
Dra. Sabina Habegger Lardoeyt \\ Observatorio CIMAS, España \\ habeggersabina@yahoo.es \\ Dra. Iulia Mancila \\ Departamento de Didáctica y Organización Escolar \\ Universidad de Málaga. España \\ imancil@uma.es
}

\section{Cómo citar / citation}

Habegger, S. y Mancila, I. (2019) "Estilos alternativos de desarrollo local: metodología utilizada para el caso de una Investigación Acción Participativa en la provincia de Málaga". OBETS. Revista de Ciencias Sociales, 14(1): 233-257. doi: 10.14198/OBETS2019.14.1.08

\section{Resumen}

El objetivo de este artículo es evidenciar la necesidad de poner en circulación experiencias de investigación que atisban una incipiente producción creativa que permite experimentar con estilos de desarrollo comunitario. Para ello, presentamos una Investigación Acción Participativa desarrollada a nivel local, concretamente en la provincia de Málaga (España) que generó un laboratorio social polifónico, confluencia de distintas voces, prácticas y formas de hacer, y cuya finalidad última fue visualizar muchas de las problemáticas sociales diarias y concretas que vivíamos los habitantes. El proceso fue protagonizado por vecinos, vecinas, junto con movimientos sociales e investigadores, quienes 
elaboramos un informe diagnóstico y los recursos metodológicos para afrontar dichas problemáticas y mejorar nuestro entorno.

Palabras clave: investigación acción participativa, ciudadanía, movimientos sociales, modelos territoriales, cartografía.

\begin{abstract}
This article's aim is to share research experiences with incipient results of innovative characteristics such as: styles of community development. We report on a Participatory Action Research experience carried out in Malaga province (Spain), which generated a polyphonic social laboratory. It's main purpose was to visualize concrete social problems that Malaga inhabitants lived on a daily basis. Neighbours, together with social movements and researchers, carried out a collaborative diagnosis report, as well as a guide to confront with the existing problems in order to solve them creatively.
\end{abstract}

Keywords: participatory action research, citizenship, social movements, territorial models, cartography.

\title{
Extended Abstract
}

This research carried out a diagnostic practice of the diverse territorial problems of Malaga province, involving a wide network of civil actors in a collective production of polyphonic, open, creative knowledge. The questions that guided this research revolve around 3 axes (urban speculation, precariousness and immigration) and are the following: to diagnose the territorial and urbanity problems of Malaga involving the existing Social Movements: to locate and make visible the focal points of conflict and social self-organization; to describe and connect resistance sources; to create networks and spaces for dialogue and communication between social movements; to interconnect, unify struggles and empower social spaces by circulating the practices of social movements as well as their own analyses; to visualize the damage / environmental and social impact, and to link the local problem with the global and the global with the local.

To answer these questions we have opted for a Participatory Action Research (PAR) to diagnose the territory and the context of the various conflicts that the current development model was causing in Malaga, involving the main affected and representing actors in this process.

We understand IAP as a type of collective research from the dialogical critical perspective, where the design and the process itself is constructed with the participation of the subjects; the situations experienced are problematized through reflective processes and various proposals that revert and / or improve these practices are developed in a participative way. This methodology is based on research techniques where conversations, analysis and social creativity workshops generate approximations "from" the context itself and "with" the protagonists (Rodríguez Villasante 2006, Martí, 2002, Alfama i Guillén, 2009). Specifically, the IAP has allowed us to tune into a common discourse respecting the peculiarity of the subgroups that composed it in a practice of democratic innovation for the improvement of our closest environment. 
In the first phase (1st year) the research design has been carried out in a shared and horizontal manner, based on face-to-face meetings for a self-diagnosis and a collective projection to become a social research laboratory. This stage involved 303 people and lasted for 12 months. The used techniques were mainly 17 interviews, 15 participant observations, SWOT workshops (Gil Zafra, 1998) and other reflection workshops like various matrixes of proposals or mappings of involved actors. A research main group: Grupo Motor (Martí, 2000) was formed, including 10 people: an architect, critical with the city models that were being developed in Malaga, a lawyer and an environmental activist especially concerned with the quality of water and the impact of new urban policies, two sociologists, two activists from social, a critical graphic designer with the mass media and the critical need of project innovations, a pedagogue interested in social movements and new styles of citizen participation and hacker psychologist with innovative formulas of political organization.

The members of the Motor Group opened a democratic space of confluence of theory and practice, in relation to different sensitive issues and the struggles carried out by the different actors. Ethically, they all were co-researchers, coparticipants of a common project that was developed in participative ways. The second phase ( 5 months) consisted of two main activities: the self-training (both methodologically and thematically) of the Motor Group and the fieldwork involving in a social cartography of the main conflicts and resistance in the province of Malaga (Pre- Report). A strategic planning has been developed collectively for the registration, systematization, categorization, analysis and production of the first reports around 3 thematic axes: urban speculation, precariousness and immigration.

Participatory planning allowed us to organize, systematize and analyse in a rigorous and creative way the enormous amount of information collected through 53 video-interviews, 6 drifts (observations during tours for a total of 14 hours), 11 conflict mapping workshops, 5 participant observations of assemblies and meetings (with the participation of 259 people) and 15 meetings of organizations, reading and analysis of documentation by the Motor Group. In this process, we have made use of digital tools, wiki, creation of collaborative web pages that allowed us to organize the material, record the decisions and show the previous reports that were generated for each axis of analysis.

In the third phase, more analytical in nature ( 7 months), the returns of the reports of partial diagnoses have been made with the participating groups. The Territorial Participative Diagnosis has been constructed in different open, public and accessible formats based on the triangulation of the information. Finally, the various initiatives and responses have been compiled, new proposals have been built and action agendas have been socialized. All this collective knowledge has been translated into different formats: a new social map (conflicts on the side A and proposals on side B), a cartographic video of the three themes (38 minutes) and a proposal guide. The participation in this stage was of 897 people. 
Finally, we can mention the generation of new emerging action cycles ( 1 year), which have allowed us to interrelate with more than 300 people (constituting working groups around the potential of cartographic productions, carrying out workshops with activists, hackers, students in a master's degree in political science and public administration officials). In this period new spaces were generated with other networks, not so related, more hybrids, which allowed us to converge in diverse productions (including self-surveys in social movements, involvement-participation with secondary schools and institutes, academic seminars or promotion of new methodologies and programs in public administration).

The Participatory Diagnosis showed a multitude of geo-referenced conflicts and resistance around the 3 thematic axes: urban speculation; immigration and precariousness. Also, more than 200 citizen proposals to solve these conflicts were generated and could be summarized as follows:

- In relation to the real estate sector, 50 land planning and agriculture initiatives.

- In relation to the social sphere, 67action of participation and networks, training, quality of life, cultures and peoples' identities, among others.

- In relation to the Environment area, 46 proposals on the new culture of water, the ecological valuation of our territory, food sovereignty, and proposals to paralyze the environmental impacts as well as strengthen their prevention.

- In relation to the Economic field, 30 proposals of tourism and employment.

\section{Introducción}

En los últimos años estamos viviendo una crisis de percepción, crisis que se apoya y se enmarca en una ciencia delineada desde el poder, con perspectivas epistemológicas concretas y sobre las que se construyen y re-construyen conocimientos esencialmente hegemónicos. Dichos conocimientos, especialmente avalados por miradas exclusivamente técnicas, vienen presentando formas muy concretas de diagnosticar y analizar los problemas sociales, ambientales y económicos de nuestras comunidades. Al mismo tiempo, este entramado, condicionado por criterios mercantilistas, avala estilos clientelares de gestión pública, influye en la toma de decisiones y legitima la implementación de políticas como medio de dominación y exclusión, afectando a nuestros territorios físicos y sociales así como configurando un modelo único de desarrollo en base a lógicas capitalistas (Habegger y Mancila 2005, Habegger 2008, 2017).

Muchas de estas prácticas se imponen y se reproducen en distintas latitudes del mundo, caracterizándose por ser insostenibles, moldeando un estilo de vida (principalmente consumista), y atentando sobremanera nuestros derechos. En definitiva, numerosos de los conocimientos creados y las herramientas de las 
que se nutren para analizar la complejidad social, parcelan la mirada (Capra, 1992), se alejan bastante de las necesidades del bien común y no generan soluciones de fondo a muchos de los problemas actuales de nuestras comunidades.

De Sousa Santos (2008) nos alerta de la neutralidad en las ciencias y nos invita a pensar en la necesidad de posicionarse y asumir una posición ética y política; construir nuevos conocimientos desde una ecología de saberes, requiere de la visibilización y amplificación de prácticas contrahegemónicas, su difusión y circulación.

En este sentido, el artículo plantea la necesidad de considerar los entresijos epistemológicos, metodológicos, políticos e ideológicos de tales prácticas e intervenciones y preguntarse acerca de si contribuyen de forma significativa a la mejora de nuestras vidas y a la creación de una sociedad más justa y sostenible para todos (Freire, 1973; Max Neef, 1986; Matus, 1995, Maturana, 1996). Consideramos imperante adentrarse en un diálogo abierto que invite a la reflexión y facilite al avance en el campo del saber científico para el desarrollo comunitario en base a los desarrollos teóricos y las evidencias empíricas desde experiencias emergentes como los laboratorios ciudadanos. Las metodologías participativas, que implican a la vez teoría y práctica, aspectos subjetivos y objetivos, perspectivas locales y globales, individuales y sociales, personales y políticas, nos adentran en distintos paradigmas de investigación (Ibañez 1992; De Sousa Santos, 2005, 2007; Borio 2004, Ganuza, Olivares, Paño, Buitrago y Lorenzana, 2010; Rodríguez Villasante 2017).

Se está acostumbrado a que la universidad juegue un papel de experto, no que se abra a un juego polifónico, incluso podemos posicionarnos críticamente en relación a los modos de gestión de las investigaciones socio-educativas y los estilos que se promueven exclusivamente desde los especialistas. Por ello, según el marco epistemológico sobre el que nos situemos (Ibáñez, 1986), se condicionan los resultados: las vivencias en ebullición podrían congelarse para describirse (paradigma distributivo-cuantitativo), o describirse y explicarse desde un plano contemplativo (paradigma estructural-cualitativo), o identificarse, analizarse y reflexionarse para provocar acciones de cambio y trasformaciones por parte de los propios actores implicados en la situación (paradigma dialógico-investigación acción participativa).

A diferencia de los diseños de investigación convencionales, donde los objetivos están acotados, la asimetría entre el investigador principal y el "objeto" de estudio está marcado por el poder (especialmente por el poder en la toma de decisiones y la gestión del proceso de estudio) y donde los resultados vienen predefinidos por las hipótesis; cada vez hay más voces que plantean otras formas de construcción de conocimiento posibles, en donde diferente saberes y experiencias valiosas confluyen con el saber tradicional a través de procesos sentidos y compartidos de reflexión y acción, donde los objetos se convierten en sujetos de acción, logrando problematizar, visibilizar, analizar, comprender 
las problemáticas comunitarias y promover estrategias colectivas de cambios y trasformaciones. Hoy en día se viene experimentando con laboratorios ciudadanos, que bien por invitación del Estado, desde el ámbito empresarial/tecnológico o por irrupción de la población autogestionada, están configurando incipientes dinámicas y estrategias de co-construcción de conocimiento en donde actores varios (universidad, sociedad civil, movimientos sociales, profesionales de la educación, comunicación u otras ciencias sociales) están inmersos en procesos y proyectos colaborativos de trasformación social (Romero-Frías \& Robinson García, 2017).

Ahora bien, según Cohen, Manion y Morrison (2000) y Kemmis y McTaggart (2005) podemos hablar de tres modelos de producción de conocimiento dentro de este último marco de compresión: modelos de investigación acción en tercera persona con un pronunciado carácter instrumental (ya que los proyectos son de una determinada duración y lo que se pretende es buscar soluciones rápidas y efectivas a un problema especifico, una situación concreta y suelen ser promovidos y gestionados por un investigador-técnico), modelos de investigación acción en segunda persona que implican una compresión profunda de las situaciones sociales y tienen un valor práctico en cuanto a la mejora de dichas situaciones y por último, los modelos de investigación en primera persona de carácter sociocrítico (los cuales evidencian y ponen de manifiesto los condicionantes estructurales y los ejes de poder asimétricos que vulneran nuestros derechos, anulan nuestra dignidad e impiden la participación, la autonomía, la libertad de acción y promueven otros campos de relación).

En algunos proyectos emprendidos, muchas veces tildados de participativos, lamentablemente distancian los posibles encuentros en torno a la mejora y el control democrático de quienes por un lado diagnostican (función que recae normalmente en técnicos/científicos especialistas), frente a quienes formulan las estrategias e implantan las medidas de "resolución" en conflictos y problemas sociales (función que suele incurrir en figuras políticas o con poder político), con respecto a quienes son intervenidos o asistidos (que suelen ser mayoritariamente el resto de la población civil). Las políticas públicas, aunque se configuran en el seno de un conjunto de redes de instituciones y grupos, y sus roles están predefinidos, promueven principalmente estilos de participación desde fórmulas de "poder-sobre" (Holloway, 2002), a través de dinámicas asistencialistas, clientelares y autoritarios (Pindado, 2005), y se trabaja de manera aislada y parcelada, acotados por rangos de poder; por el contrario, en este entramado de diversos de agentes (políticos, técnicos y ciudadanos), los estilos de participación podrían impulsarse con características de proximidad y visión interrelacionada (Canal, 2017).

En este sentido, el papel de la investigación social podría configurarse como otra forma de hacer pedagogía del territorio de manera que se conformaran redes de construcción de conocimiento más allá de las protestas, incorporando 
vivencias invisibles, voces silenciadas, resistencias y prácticas emergentes en los espacios colaborativos de análisis y propuestas para la acción transformadora desde la colectividad. Por ello, se requiere de mecanismos y metodologías alternativas, innovadoras, para que los procesos de construcción de conocimiento puedan resolver de manera más democrática e inclusiva la multitud de problemas socioeconómicos que impactan en la ciudadanía, en los territorios y construyan conocimiento más creativo. Esta nueva epistemología, se incluye la participación de la ciudadanía, no solo en la problematización de los asuntos públicos sino en la manera de revertirlos y resolverlos (Azcueta, 2004; Rodríguez Villasante, 1994, 1995, 2002; Hernández, 2002). El reto se presenta en configurar laboratorios sociales polifónicos, donde suenen simultáneamente múltiples voces, con la intención de rescatar, conocer y poner en sintonía prácticas con elementos más o menos comunes, enriquecer el análisis y promover el entendimiento de la realidad compleja de nuestro entorno. Asimismo, el laboratorio social polifónico tiene como objetivo crear sinergias entre los diversos discursos y las acciones ciudadanas, lo que requiere de posicionamientos permeables, críticos a los modelos territoriales que se vienen instaurando desde políticas mercantilistas globales y locales y con capacidad de abrirse y ejercitar propuestas innovadoras en aras del bien común.

\section{Metodología}

Concretamente esta investigación consiste en una práctica diagnóstica de las diversas problemáticas territoriales y urbanísticas de la provincia de Málaga, involucrando a una amplia red de actores (investigadores, miembros de diferentes movimientos sociales, vecinos, representantes de la sociedad civil) en una producción colectiva crítica de conocimiento de carácter polifónico, cartográfico, abierto, creativo. Las preguntas que han guiado esta investigación giran sobre 3 ejes (especulación urbanística, precariedad e inmigración) y son las siguientes:

- Diagnosticar la realidad malagueña desde los Movimientos Sociales (icuáles son los temas sensibles, las denuncias, los anuncios, las redes, las manifestaciones en nuestra provincia? ¿cuáles son las experiencias y las expectativas de los movimientos?, ¿cuáles son las prácticas insostenibles y en qué nos afectan?, ¿a qué se deben los conflictos (causa/s) y qué efectos provoca en el territorio (consecuencias en los físico y en lo social)?, ¿qué derechos se vulneran? ¿qué leyes o normativas se incumplen?, ¿qué actores intervienen en las decisiones? ¿qué empresas están relacionadas?, ¿qué información hay al respecto? ¿existen discursos técnicos, académicos, sindicales que sostengan estas miradas?). 
- Localizar y visibilizar los focos de conflicto y auto-organización social (¿qué colectivos, grupos de personas o prácticas que podemos geo-refenciar en el mapa de la provincia?, ¿qué informantes clave nos puede ayudar a identificar los conflictos y los espacios de organización social en resistencia?, ¿quiénes son, dónde están, qué dicen, qué hacen, cómo se organizan, con quién?, ¿cuáles son las tendencias).

- Describir y conectar las bolsas de resistencia (¿qué discursos de resistencia existen en torno a los conflictos?, ¿qué denuncian y cómo lo justifican?, ¿quiénes reivindican cuestiones similares en el territorio?, ¿cómo se relacionan?, ¿qué similitudes/diferencias existen con otros nodos de resistencia?).

- Interconectar, unificar luchas y potenciar espacios sociales haciendo circular las prácticas de los movimientos sociales así como los análisis propios (¿qué discursos existen sobre los conflictos y qué interconexiones tiene en lo local?, ¿qué procedimientos, planes, proyectos comunes se desarrollan en torno a los conflictos?, ¿cómo se pueden unificar distintos movimientos sociales para conseguir una alternativa viable?).

- Visualizar el daño/impacto ambiental y social (¿Qué información producen las resistencias (datos, textos, imágenes y otros materiales)? ¿están sistematizadas? ¿es accesible para toda la ciudadanía?).

- Enlazar la problemática local con lo global y lo global con lo local. (¿cómo y con quiénes se toman las decisiones en proyectos sobre el territorio?, ¿existen actores o lógicas coincidentes, interdependientes?, ¿cómo se interrelaciona con lo global?).

Para dar respuestas a estas preguntas hemos apostado por una Investigación Acción Participativa (IAP) de manera que nos permitiera diagnosticar el territorio y profundizar en el contexto de los diversos conflictos que el modelo de desarrollo actual estaba causando, involucrando a los principales afectados y representando dichas realidades en códigos accesibles.

Entendemos por IAP un tipo de investigación colectiva desde la perspectiva crítica dialógica, donde el diseño y el proceso mismo se construye con la participación de los sujetos; se problematizan las situaciones vividas mediante procesos reflexivos y se elaboran participativamente propuestas que reviertan y/o mejoren sus prácticas. Esta metodología se nutre de técnicas de investigación donde las conversaciones, el análisis y los talleres de creatividad social generan aproximaciones "desde" el propio contexto y "con" los actores protagonistas (Rodríguez Villasante 2006; Martí, 2002; Alfama i Guillén, 2009). Ejemplos de investigaciones con esta metodología se han destacado en trabajos históricos de Freire (1973), Ander-Egg (1990), Marchioni (1992), Fals Borda (1990), y 
especialmente las prácticas promovidas por el Colectivo Iepala y Cimas en España, así como la sistematización de éstas últimas a través de la colección de Construyendo Ciudadanía, de la editorial Viejo Topo, muestran multitud de experiencias locales y más actuales en el campo de la salud, educación, empleo, autogestión, cultura, gestión pública, etc.

Concretamente, el proceso puesto en marcha y en sus distintas fases permite sintonizar un discurso común respetando la peculiaridad de los subgrupos que lo componían en una práctica de innovación democrática para la mejora de nuestro entorno más cercano.

En la primera fase ( 1 año) se realiza la construcción del diseño de investigación de manera compartida y horizontal. Será a partir de encuentros presenciales en un espacio de foro social (autodiagnóstico), donde se implican 303 personas (véase la Tabla 1 más abajo) y donde se desarrollan encuentros reflexivos con ciertos intereses y necesidades comunes. Las técnicas empleadas son principalmente 17 entrevistas, 15 observaciones participantes, taller DAFO -Debilidades, Amenazas, Fortalezas y Oportunidades (Gil Zafra, 1998) y otros talleres de reflexión (matrices de propuestas, mapeos de actores), análisis mediante informes y devoluciones correspondientes. Sobre una de las devoluciones empieza a nacer el diseño participativo hasta constituirse un Grupo Motor (Martí, 2000), que conformando por 10 personas, se compromete a emprender la investigación sobre el territorio focalizando el interés sobre conflictos y resistencias en 3 ejes, concretamente el grupo lo componen: un arquitecto crítico con los modelos de ciudad que se vienen desarrollando y con proyecto de final de carrera, un abogado y activista medioambiental preocupado especialmente por la calidad del agua y el impacto de las nuevas políticas urbanísticas, dos sociólogos, uno activista de incipientes estilos políticos desde los movimientos sociales y otro interesado de acompañar esta experiencia, una diseñadora gráfica crítica con los medios de comunicación masiva y la necesidad crítica de proyectar innovaciones, tres estudiantes de comunicación con creatividad y deseos de experimentar un proceso de investigación colectiva, una de ellas activista de un centro cultural okupa, una psicopedagoga con proyecto de tesis doctoral en los movimientos sociales y nuevos estilos de participación ciudadana y un psicólogo activista cultural y hacker con fórmulas innovadoras de organización política.

Los integrantes del Grupo Motor abren un espacio democrático de confluencia teoría y práctica, nutrido por los temas sensibles y las luchas protagonizadas por los diferentes actores, se posicionan éticamente al considerarse como coinvestigadores, co-participantes de un proyecto común que se gesta participativamente, se hace propio y logra convertirse en un nuevo sujeto colectivo (Véase Fig.1). 
Figura 1. Fases de la IAP

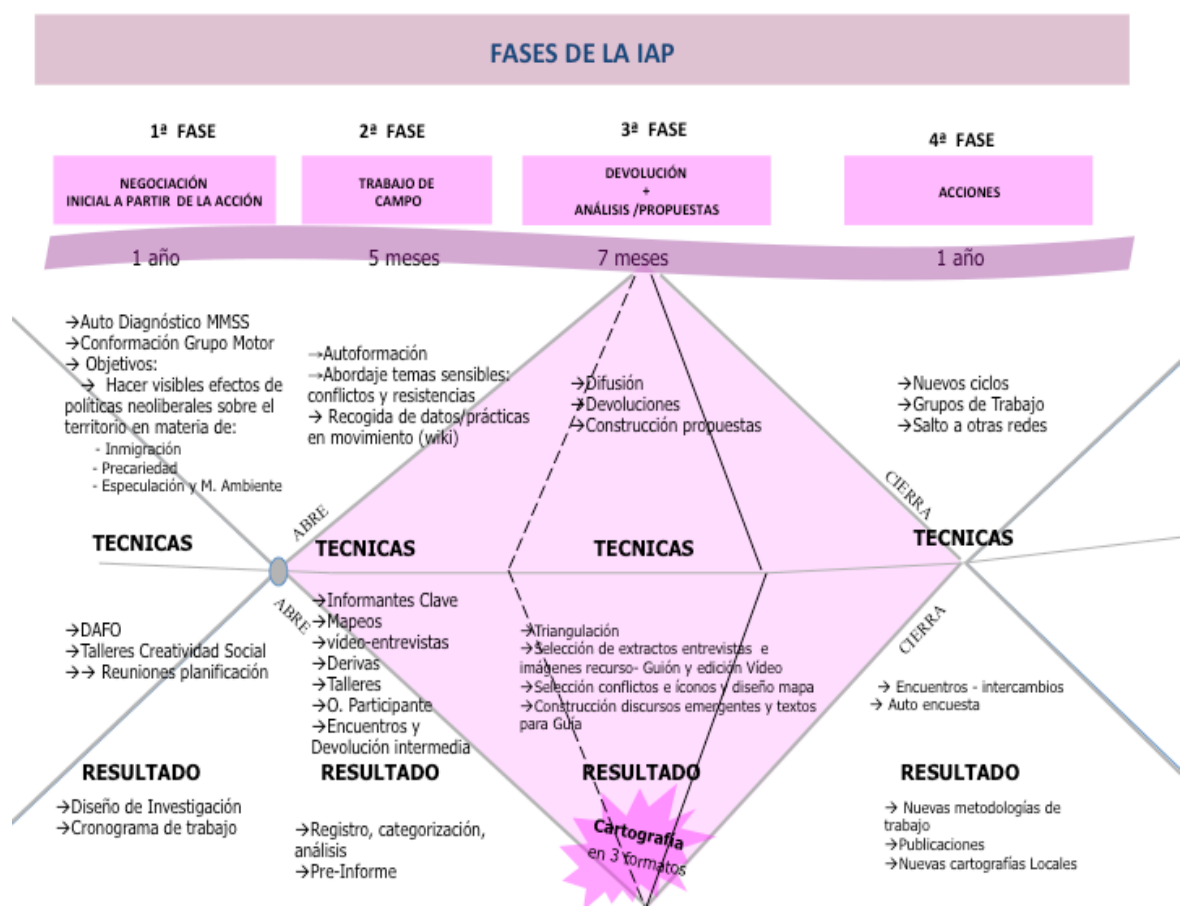

Fuente: Elaboración propia

La segunda fase (5 meses) consiste en dos principales actividades: la autoformación (metodológica y temática) del Grupo Motor y el trabajo de campo para la realización de una cartografía social de los principales conflictos y resistencias en la provincia de Málaga (Pre-Informe).

La cartografía social permite a las comunidades conocer y construir un conocimiento integral de su territorio para que puedan elegir una mejor manera de vivirlo. Es una propuesta conceptual y metodológicamente novedosa que hace uso de instrumentos técnicos y vivenciales (Habegger y Mancila, 2005: 33).

Los primeros pasos se hacen desde las redes concretas de activistas: con apoyatura en la propia comunidad de conflicto como en otros actores con perfil técnico.

Para ello, se elabora de forma colectiva una planificación estratégica para el abordaje territorial: asumir el registro, la sistematización, la categorización, 
análisis y producción de los primeros informes en torno a los 3 ejes temáticos: especulación urbanística, precariedad e inmigración.

La planificación permite que el Grupo Motor asumiera de forma rigurosa y creativa la ingente cantidad de información recogida (53 vídeo-entrevistas, 6 derivas -observaciones durante recorridos por un total de 14 horas-, 11 talleres de mapeos de conflictos, 5 observaciones participantes de asambleas y reuniones -con la participación de 259 personas- y 15 encuentros de organizaciones), se formara mediante lectura y análisis de documentación específica ( AA.VV., Cimas, 2009) y se atuviera a una serie de tiempos donde producir pre-informes parciales y sincronizarse para una producción conjunta de diagnóstico territorial (apoyándose de herramientas digitales como una wiki).

En la tercera fase ( 7 meses), se triangula y se empieza a analizar, se construye el Diagnóstico Participativo Territorial y se socializa a la ciudadanía (devoluciones a los colaboradores). El diagnóstico se apoya de un formato cartográfico (abierto, público y accesible), concretamente sobre un mapa, un vídeo y un libro-guía. Se visualizan los conflictos, las resistencias, se recopila las diversas iniciativas y respuestas construidas (batería de propuestas emergentes y agenda de acciones). Concretamente el mapa en papel geo-referencia conflictos en la cara A y propuestas en la cara B, el vídeo de los tres ejes (de 38 minutos) evidencia el territorio a través de imágenes recurso y testimonios en primera persona, la guía (un libro de 184 páginas) deja huella del proceso con imágenes, extractos de entrevistas, textos propios de las resistencias y fichas de movimiento. La participación en esta etapa es de 897 personas.

Finalmente, podemos mencionar un punto de inflexión que abre nuevos ciclos de acción emergente ( 1 año), donde nos interrelacionarnos con unas 300 personas más (se crean grupos de trabajo en torno a las potencialidades de las producciones cartográficas, jornadas, talleres con activistas y hackers por un lado y con estudiantes en máster de ciencias políticas o funcionarios de la administración pública por otro). En dicho periodo se empieza a crear nuevos espacios y saltos a otras redes, no tan afines, más híbridos (incluyendo auto encuestas en los movimientos sociales, implicación-participación con escuelas e institutos de secundaria, devoluciones en jornadas académicas, o impulso de nuevas metodologías y programas en la administración pública etc.). En la siguiente Tabla se sintetiza los instrumentos de recogida de información y los participantes: 
Tabla 1. Instrumentos de recogida de información y participantes

\begin{tabular}{|c|c|}
\hline \multicolumn{2}{|l|}{$\begin{array}{ll}1^{\mathrm{a}} \text { FASE }(1 \text { año }) & \text { Negociación Inicial } \\
\end{array}$} \\
\hline TÉCNICAS & $\begin{array}{l}\text { CANTIDAD DE PARTI- } \\
\text { CIPANTES }\end{array}$ \\
\hline 17 Entrevistas (individuales y colectivas) & 20 \\
\hline 15 Observaciones Participantes & 193 \\
\hline Diario de campo de la investigadora & - \\
\hline Análisis datos y 10 documentos & - \\
\hline Fichas de Movimientos Sociales (MMSS) y Organizaciones & 8 \\
\hline 1 Taller D.A.F.O. & 13 \\
\hline 1 Taller Matriz Soluciones & 13 \\
\hline 1 Mapeo de actores (con Informante Clave -I.C.-) & 1 \\
\hline 3 Talleres de Devolución & 45 \\
\hline 2 Documentos Auto Diagnóstico & 10 \\
\hline Sub Total & 303 \\
\hline \multicolumn{2}{|l|}{$\begin{array}{l}2^{\mathrm{a}} \text { FASE ( } 5 \text { meses) } \\
\text { Laboratorio ciudadano - Grupo Motor: Trabajo de campo }\end{array}$} \\
\hline Grupo Motor (G.M.) & 10 \\
\hline 3 Mapeo de I.C desde el G.M. & 11 (I.C.) \\
\hline 53 Vídeo Entrevistas & 53 \\
\hline 5 Observaciones Participantes desde el G.M. & 119 \\
\hline 15 Fichas de los MMSS y Organizaciones & 15 \\
\hline 25 Análisis de documentos & - \\
\hline 17 Reuniones periódicas del G.M. & - \\
\hline 10 Mapeos de conflictos & 39 \\
\hline 6 Derivas & 12 \\
\hline Sub Total & 259 \\
\hline \multicolumn{2}{|l|}{$\begin{array}{l}\text { a FASE (7 meses) } \\
\text { Devolución: Análisis y propuestas }\end{array}$} \\
\hline 9 Talleres de socialización & 273 \\
\hline 7 Talleres de Devolución & 624 \\
\hline Sub Total & 897 \\
\hline \multicolumn{2}{|l|}{$\begin{array}{l}4^{\mathrm{a}} \text { FASE }(1 \text { año }) \\
\text { Acciones }\end{array}$} \\
\hline $\begin{array}{l}7 \text { Grupos de trabajo (cartografías con metodologías participativas aplicadas a diagnósti- } \\
\text { cos institucionales, barriales - comunitarios) }\end{array}$ & 300 \\
\hline Sub Total & 300 \\
\hline TOTAL PARTICIPANTES & 1.759 \\
\hline
\end{tabular}

\section{Fuente: Elaboración propia}




\section{Resultados}

Consideramos que el hecho de disponer de un producto cartográfico participativo (saberes, discursos, prácticas) y en multi-formatos tangibles y virtuales como el mapa, el vídeo, el libro-guía así como la sistematización del proceso en un wiki, concatena nuevas acciones, especialmente a raíz de su circulación con la devolución y socialización; produciendo incipientes ciclos sinérgicos con otros actores muchos más diversos: saltando de redes "afines" por las que nos veníamos moviendo a otras más "diferentes" (Martín Gutiérrez, 1998, 2001). Es decir, esta investigación, mediante la metodología de IAP y sus varios ciclos, permite construir un Diagnóstico Participativo Territorial, evidenciar una multitud de conflictos geo-referenciados y resistencias en los 3 ejes, como también construir baterías de propuestas, análisis y agendas de acciones de impacto. A continuación, presentamos algunos de los resultados según los objetivos de los que partíamos para construir el diagnóstico, así como resultados surgidos desde el análisis y la batería de propuestas emergentes. Finalmente compartimos algunos resultados en torno a la autoevaluación del Grupo Motor y los desbordes que llevan nuevas líneas de acción cartográfica y local.

\section{Resultados desde el Diagnóstico Participativo Territorial}

\section{a) Especulación urbanística}

El eje de Especulación y Medio Ambiente gira en torno al desarrollo incontrolado después de haber saturado la primera línea de costa, lo que empuja la especulación del territorio hacia el interior de la provincia. Un modelo de desarrollo con impacto insostenible, tanto ambiental (paisaje, recursos hídricos, sobreexplotación de canteras, desaparición de fauna y flora) como social (aumento exponencial de la población, desaparición de la cultura y producción de cada lugar, abandono de cultivos, modelo productivo basado en el turismo). Un modelo turístico masivo en base a un "marca" que termina de "vender" el territorio y donde la población autóctona apenas tiene poder en la toma de decisiones y solo sufre sus consecuencias (desplazamiento y expulsión de su población, pérdida generacional de empleos y parón en el relevo generacional en el campo, despoblación del centro de la ciudad y el centro de los pueblos, restricciones o sin acceso a agua potable a sus habitantes durante la mayor parte del año, infraestructuras de pagos como las autopistas sin beneficio alguno para los pueblos del interior, gestión privatizada de los servicios, etc.).

Numerosos actores identifican los temas sensibles que les afectan en su día a día: tendencias urbanizadoras, campos de golf y urbanizaciones, hoteles de nueva construcción, transformación del territorio al interior de la provincia, 
impactos medios ambientales y sociales, agua, canteras, presas y trasvases, casas ilegales, conexiones (autopistas y aeropuertos, AVE), invernaderos, centrales térmicas y resistencias.

\begin{abstract}
"Actualmente en todo Málaga, independientemente del color político de sus alcaldías, se está implementado en modelo de crecimiento marbellí. Este prototipo parte de un pensamiento único de desarrollo, y se materializa en un crecimiento urbanístico sin planificar, incontrolado, con una elevada cantidad de construcciones ilegales, toleradas por ayuntamientos, por la propia Junta de Andalucía, justificándose hacia la población con promesas de puestos de trabajo, la mayoría temporales y precarios. Este modelo, mediante sus urbanizaciones y campos de golf, trae consigo una masiva destrucción del medio ambiente: en la Costa del Sol no queda prácticamente franja de costa liberada de ese cáncer que también está a punto de colonizar el interior, como la zona de Antequera donde en el encinar protegido de la Pedrizas se proyectan cuatro campos de golf. Otro ejemplo ilustrativo: Coín, con un campo de golf sobre el acuífero que abastece al municipio..." (Entrevista J. M. C. miembro de Ecologistas en Acción- Málaga).

"En Antequera, Coín, Ronda, Gaucín, Jimena de la Frontera y en el resto del territorio de la provincia existen los mismos problemas que se padecen aquí: el intento de terminar con la idiosincrasia de los pueblos, sus particularidades, su agua, su paisaje...para obtener unos rendimientos económicos y de convertirlo todo en una mercancía. Pero hay cosas que están fuera del comercio y una de ellas es la autogestión. No nos vendemos...El proyecto para Villanueva del Rosario de 900 viviendas y un campo de golf no se puede hacer contra la voluntad de los 1330 vecinos (de un total de 3000 aproximadamente) que han firmado en contra del mismo" (Entrevista F. P. V, Coordinadora por la Defensa del Medio Ambiente Rural y Litoral).
\end{abstract}

Todo ello se georeferencia sobre la cara A del mapa: los conflictos y algunas descripciones como los proyectos urbanísticos que estaban desarrollándose y planificándose en el territorio (concretamente miles de viviendas y mediante iconos de macro urbanizaciones aprobadas y/o en construcción especialmente en pueblos del interior de la provincia), así como los hoteles en primera línea de costa y pueblos del interior y centros comerciales (bajo el icono de "Hoteles" aprobados o en construcción y "catedrales de consumo" respectivamente). También se geolocalizan proyectos los campos de golf y los que están en proyectos.

No obstante, algunos de los temas identificados no logran atajarse en profundidad por la temporalidad de la investigación y la necesidad de un cierre y devolución (concretamente presas, trasvases, AVE, invernaderos y casas ilegales). Por el contrario, algunas otras ideas no predefinidas surgen y son abordadas (análisis de PGOU, decretos, cambios en calificaciones de terreno aprobados en plenos municipales, estudios medio ambientales, convenios y ley del suelo). En cuanto al análisis de los procedimientos administrativos se identifican cómo los criterios técnicos, los planes de ordenación urbana, la 
externalización del servicio público y la inexistencia de programas públicos o la composición de plenos municipales posibilita la proliferación de este modelo especulativo.

Para entender la magnitud de los conflictos urbanísticos, se logran identificar impactos concretos como el desborde de las infraestructuras locales y la desaparición de terrenos originalmente agrícolas y forestales, provocándose talas indiscriminadas de más de 1.200 encinas, entre otras especies, la contaminación del agua, la modificación de las señas de identidad de los pueblos del interior, tanto en la infraestructura (amasijos de viviendas modernas y urbanizaciones en convivencia con las casas blancas típicas los pueblos, reconversión de suelos rústicos a suelos urbanizables, degradación del patrimonio), la pérdida de oficios y costumbres (adaptando la demanda del turista a la reformulación cultural y políticas de los municipios del interior) o la dificultad en el acceso a la vivienda (especialmente para jóvenes frente a la especulación promovida por el capitalismo posmoderno, privatización del suelo público), entre otras.

“(...) el 69\% de las muestras analizadas de acuíferos de la cuenca Sur han arrojado niveles de nitratos por encima de los $50 \mathrm{mg} / \mathrm{l}$ que establece la OMS como límite para aguas potables...A más contaminación de las aguas, más cantidad de cloro para su potabilización. Y se sabe que el cloro unido a determinados compuestos orgánicos que llevan las aguas contaminadas, dan lugar a los trihalometanos, que son substancias potencialmente cancerígenas, mutógenas y teratógenas. (Entrevista F.P.V. Coordinadora por la Defensa del Medio Ambiente Rural y Litoral).

\section{b) Inmigración}

La inmigración es uno de los ejes de conflicto que atraviesa la provincia. Desde el Estado se asiste aun intento de regularización de los flujos migratorios siempre incompleto, desbordado. Lo que se llama "régimen de fronteras" es un ejercicio sin éxito para controlar y gestionar estos flujos de personas trabajadoras, en aquellos nichos laborales abandonados por trabajadores autóctonos precarios. Por lo tanto, el eje de inmigración está íntimamente ligado al trabajo y la precariedad. Además, como otro dispositivo de control, el estatuto legal del inmigrante, determina su valor en el mercado de trabajo y su ausencia, una violación de los derechos básicos y causa de la discriminación múltiple y la exclusión social.

Gracias a los participantes en la investigación a nivel de colectivos o nivel personal, se han identificado las siguientes tensiones y problemáticas: espacios de flujos migratorios -cuencas, sectores productivos-, condiciones de trabajo, dificultades de acceso a la vivienda, los espacios de control y contención y disciplina de los flujos migratorios. 
“...los marroquíes aquí no pueden trabajar sin un mediador. La prueba es que hay mucha gente con papeles y que está en la calle, todo el día buscando trabajo y no lo encuentra por ser marroquí, sobre todo, después del 11 de marzo y con lo de Isis...Hay gente que sufre más aquí que en su país, no tienen medios de vida, ni donde dormir, ni nada, gente que ha cogido muchas enfermedades, en la agricultura los tóxicos te lastiman la piel. Hay muchos marroquíes y africanos que no tienen nada, duermen en la playa, todos los días, eso está pasando a 15 minutos de aquí." (Entrevista inmigrante marroquí, residente en Málaga).

También se identificaron las resistencias, asociaciones y especialmente personas no organizadas.

"Bueno, nuestra asociación trata de aglutinar a las diferentes culturas porque Marbella y su área de influencia acapara a más de treinta y cinco nacionalidades diferentes; la asociación juega un papel importante para los propios inmigrantes y no solamente eso; la propia sociedad marbellí puede beneficiarse. Te voy a dar un ejemplo de lo que está pasando: en el colectivo ucraniano, por ejemplo, y ruso, tenemos profesores de música de muy alto novel y aquí estamos escasos". (Entrevista representante Asociación Inmigrantes).

En el decurso de la investigación surgen temas sensibles emergentes, originalmente no contemplados como el de las personas inmigrantes en calidad de trabajadoras cuidadoras. En este sentido las propias redes que se identifican al inicio de la investigación se entrelazan con nuevos contactos y nuevas realidades que necesitan ser visibilizadas también.

Asimismo, se analiza la proliferación de internamientos y privación de derechos a inmigrantes en los CIE -Centro de Internamiento de Extranjeros y se geo-referencian de zonas de control y represión abusiva de inmigrantes, vulneración de derechos a la vivienda, al trabajo y vacíos institucionales.

"El problema del CIE de Capuchinos es que no siempre cuenta con un trabajador social- ahora mismo, por ejemplo, no hay ninguno- por lo que las personas que hay allí tienen una situación de completa exclusión y aislamiento, no hay nadie que monitoree las condiciones de este lugar...Tampoco cuentan con servicios médicos permanentes para atender a la población interna. No existe un equipo de mujeres para cuidar o asistir a las mujeres... Hay problemas de masificación, las celdas nos son individuales y además se trata de un centro que está custodiado, interior y exteriormente, por funcionarios de policía...Esto puede dar pie a todo tipo de abusos, como el caso de la violación ocurrido contra una chica brasilera, o la barbaridad de meter a 36 personas en una celda, como ocurrió en el incendio de la comisaria de Málaga donde murieron siete inmigrantes". (Entrevista abogado ONG Andalucía Acoge).

Además, la canalización de responsabilidades públicas a intervención exclusiva de ONG's es otra de las características de la provincia identificadas por nuestros participantes. 
“...el Ayuntamiento de Málaga al inicio reunió todas las organizaciones para ver qué problemática hay, e intentar poner solución, luego ha ido empeorando. Otro modelo se ha basado en apoyar a organizaciones concretas que prestan servicios y convertirlos en oficinas del ayuntamiento. Para mí la administración que más tiene que decir al final sobre estos procesos, es la local. Si la administración local se emperra en que no da vivienda de protección, en no permitir el punto de encuentro a los inmigrantes, o en diferencias la atención a los inmigrantes, posiblemente esto produzca más conflictos" (Entrevista trabajador social ONG para la integración de las personas inmigrantes)

Estos aspectos se vuelcan en el Libro-Guía y otros en el vídeo. El mapa, en su cara A logra identificar con iconos y zonas de intensidad la conflictividad del eje.

\section{c) Precariedad}

La precariedad es un fenómeno complejo que va más allá del ámbito laboral y afecta la misma capacidad de decisión y libertad sobre la propia vida y actividad social, generando una situación de impotencia, malestar, sometimiento y alienación de las personas.

"He trabajado en tres empresas distintas, y la verdad es que fueron lamentables: desde no respetar horarios, no respetar las horas pactadas anteriormente, condiciones que no van de acuerdo ni a la propuesta ni a la capacidad personal... Te podría contar muchos ejemplos de falta de respeto en todos los sentidos. Hay una sensación de que uno no tiene derecho al reclamo y dentro de la propuesta laboral se incluye el maltrato, usar tu tiempo y tu vida como si fuera propiedad del empresario" (Entrevista extrabajadora Pasta City).

Varios de los entrevistados coinciden en

"que es una situación insuficiente para el bienestar de las personas" (Entrevista trabajador de Telefónica).

¿En qué consiste este fenómeno? El mismo entrevistado nos iba dando pistas:

"Hay factores que son indicios de esta precariedad: temporalidad, desregulación, contratos basura, un salario mísero que no te da ni para un alquiler de una vivienda" (Entrevista trabajador de Telefónica).

Al seguir conversando con trabajadores del sector de los servicios (limpieza, telemarketing, servicios sociales, sector de hostelería, ferroviario, construcción o sector público) se identifican temas sensibles como: focos de precariedad laboral, índice de precariedad/temporalidad, características del trabajo, incumplimiento de convenios, subcontratación, en definitiva los retazos de las denominadas "vidas precarias" en una sociedad del miedo y la fragmentación social. Múltiples testimonios nos hablan sobre la flexibilidad laboral, condiciones en 
la siniestralidad laboral, tanto desde el sector servicios, sector público y subcontrataciones.

Por otro lado, la reflexión colectiva en base a la resistencia de muchos actores, posibilita identificar las causas, los agentes responsables y los entramados especulativos que producen una economía marginal (formados por empresas, actores políticos, instituciones, promotoras, inmobiliarias, partidos políticos, empresas de servicio y trabajo temporal, telecomunicaciones), así como los pulmones sociales en resistencia, tanto asociaciones como personas sin organizar que componen el discurso de la denuncia y atisban itinerarios de acción colectiva (huelga, movilizaciones por la mejora en la conflictividad laboral en el marco de convenio), etc.

"Las mismas condiciones de miseria, esa precariedad misma, hace que la gente se movilice, porque ganar 4 euros la hora trabajando para una multinacional que en esa hora gana miles de millones de euros en toda el mundo, de alguna manera te hace que te rebeles..." (Entrevista teleoperador).

Esta información se vuelca en los 3 formatos, mediante micro informes, transcripciones de entrevistas y acompañamiento en manifestaciones y asambleas. Además de los tres ejes del conflicto, destacamos aspectos de interés en cada uno de los 3 formatos utilizados para presentar los resultados.

En el caso del mapa, se logra cubrir parte de los objetivos relacionados con la identificación, localización, visualización y descripción de los conflictos (especialmente la cara A y mediante iconos, ventanas de datos y zonas de intensidad) así como también identificar los espacios de auto-organización social (mediante la geo-referencia de "Movimientos y redes en defensa de sus formas de vida, creaciones y resistencias. Pulmones Sociales") y los mapeos de actores con las que el Grupo Motor se retroalimentó (cara A y B respectivamente).

Las cuestiones más descriptivas del Diagnóstico cartográfico se vuelcan en la cara B (mediante diagramas que surgen de la categorización de la información, ventanas y redacción de micro informes que detallan los procesos de conflicto y resistencia, causas y efectos, entramados de actores, proyectos e intervenciones). Y se posibilita evidenciar también los procesos en movimiento, las acciones de las resistencias, algunas de las propuestas de la batería como futuras agendas de encuentro y dinamización.

El vídeo por su parte resulta un formato que posibilita incorporar voz y rostros a los relatos de denuncia, así como incorporar una dimensión subjetiva, imágenes, recursos de los paisajes y las realidades denunciadas; algunas animaciones permiten generar un efecto comunicativo importante como al mismo tiempo concatenar los 3 ejes.

Finalmente, el libro - guía permite socializar muchos extractos de entrevistas con migrantes, testimonios de trabajadores, vecinos y ecologistas, análisis a través de textos construidos durante la investigación y textos críticos 
elaborados por expertos comprometidos, lo que permite entrelazar la problemática global con la realidad local, contextualiza, explica y detalla los conflictos (a través de aportes de especialistas, economistas, físicos, profesores, sindicalistas, arquitectos, presidentes de asociaciones de vecinos, estudiantes, abogados, etc.), las fichas de movimientos con contactos terminan de ofrecer cauces de comunicación con las resistencias; y la publicación en copyleft también posibilita reproducir el material bajo dicha licencia.

A continuación, en el marco de acciones construidas en el proceso y desde varias perspectivas, mostraremos sintéticamente a modo de ejemplo la batería de propuestas:

\section{Resultados desde la Batería de Propuestas}

a) Desde los ejes de investigación y con carácter de temáticas concretas surgieron más de 200 propuestas ciudadanas que se podrían resumir de la siguiente forma:

- 50 iniciativas en relación al ámbito urbanístico propiamente dicho, lo que comprenden propuestas desde la planificación del territorio y la agricultura.

- 67 iniciativas relativas al ámbito social, con acciones desde la participación y las redes, la formación, la calidad de vida, las culturas y las identidades de los pueblos, entre otras.

- 46 propuestas en relación al ámbito del Medio Ambiente con propuestas sobre la nueva cultura del agua, la valoración ecológica de nuestro territorio, soberanía alimentaria, y propuestas para paralizar los impactos medioambientales así como fortalecer su prevención.

- 30 ideas referentes al ámbito Económico, con propuestas desde el turismo y el empleo.

b) Desde el ámbito de la comunicación, la difusión de resultados y la propulsión de investigaciones sociales participativas y laboratorios sociales se propuso proseguir con el mapeo de redes sociales, tejer redes de alianzas desde proyectos comunitarios, fortalecer los vínculos estratégicos (redes de afines, incorporar diferentes y acercar ajenos), fomentar el movimiento ciudadano y trabajar desde redes de apoyo, organizarse mejor desde la sincronización de varios tiempos y estilos de participación, coordinar actividades de visibilidad bajo un calendario común en toda la provincia, realizar mesas redondas auto gestionadas: Encuentros en Movimientos (temáticos) e itinerantes por toda la provincia, etc. 
c) Desde la evaluación del propio Grupo Motor se generaron 4 líneas fuerza para un Plan de Acción Integral (Rodríguez Villasante, 2006) en torno a la formación, impulso de una segunda fase de Investigación Acción Participativa, usando las posibilidades de las nuevas tecnologías y las redes sociales y un proyecto de comunicación alternativa desde los Movimientos Sociales.

En cuanto a las agendas creadas, desde los diversos ejes como de los entresijos que resurgieron a partir de las devoluciones por toda la provincia e incluso a nivel regional y nacional, se generaron acciones y nuevos grupos de trabajo, nuevas cartografías locales, workshops universitarios en distritos de la ciudad y pueblos del interior, nuevos ciclos de IAP del Grupo Motor con Institutos de Secundaria, fortalecimiento de redes de ecologistas en una coordinadora provincial, plataforma de Inmigrantes, nuevas investigaciones en centros sociales autogestionados e implicación en procesos de democracia participativa en varios municipios del interior.

\section{Discusión y conclusiones}

Sin duda, la propia dinámica de este laboratorio social polifónico llegó a desbordar la propuesta inicial, reconstruyendo los discursos desde las necesidades y conflictos comunes, creando un conocimiento compartido con actores diferentes y, estratégicamente, fortaleciendo la investigación como medio para visibilizar problemáticas territoriales, nuevas cartografías locales y proponer alternativas de cambio, en definitiva, nuevos itinerarios de acción que se abrían y continuaban sus trayectos. Al mismo tiempo, fue el espacio abierto de confluencia de las voces y los rostros de aquellas personas que muchas veces están ausentes en el discurso oficial y la agenda de los nuevos modelos de desarrollo y gestión pública de nuestras comunidades. El laboratorio social polifónico permitió registrar, sistematizar y visibilizar muchas denuncias y resistencias y se convirtió en un espacio ciudadano desinstitucionalizado, alternativo y propositivo, promoviendo una "democracia activa de intervención" (Presno Linera, 2014).

Más allá de enumerar y explayarse en los factores que generaron sinergias, se podría decir que fue la confluencia de varios aspectos lo que permitió dibujar un proyecto social común, innovador, de co-construcción de conocimiento para un nuevo estilo de desarrollo comunitario.

Sobre todo, el deseo de experimentar desde los movimientos sociales, aprender, compartir y generar capacidades y recorridos varios, como al mismo tiempo la humildad manifiesta y la actitud necesaria para relacionarse de igual a igual y aprender unos de otros. Asimismo, la propuesta formal desde el marco de la academia, como proyecto de tesis doctoral de la Facultad de Ciencias de 
la Educación de la Universidad de Málaga, resultó fundamental en promover esta experiencia. La metodología propuesta de Investigación Acción Participativa creaba un marco horizontal, un modelo abierto a construir conocimiento donde el diseño no venía predefinido, sino que se construiría con todos los participantes. El hecho que hubiera un proyecto colectivo de investigación optimizó y dinamizó lo que en otras ocasiones se había demostrado como algo inédito, posible, pero con impulsos intermitentes desde los movimientos sociales que mostraban una desconfianza creciente hacía los partidos y dirigentes políticos, pero no terminaban de sincronizarse en una tarea compartida (Presno Linera, 2014). El haber desarrollado una negociación inicial, de manera presencial, próxima y con metodologías participativas, dio lugar a que se saltara de enfoques individuales, así como disgregados -característicos de aquellas organizaciones sociales - a generar un proceso de apropiación colectiva.

Esta tarea conlleva a diseñar estratégicamente el tipo de participación y los medios a través de los cuales se implique la ciudadanía. En este sentido se complementó la dinamización de los espacios reales con los virtuales. Los modelos organizativos requieren tanto de espacios virtuales, pero también de lo tangible, del encuentro, la discusión, el diálogo y la construcción colectiva. El uso de las redes sociales y las herramientas virtuales facilitaron la aceleración en determinados momentos del proceso, como por ejemplo agilizando el registro y la visualización compartida y volviendo a los espacios de trabajo mucho más operativos.

Un tercer aspecto o factor a tener en cuenta en el buen desarrollo de esta experiencia fue la financiación del proyecto en parte, ya que cubría únicamente gastos de traslados para el trabajo de campo, la edición de un material audio visual y la publicación de los resultados en diversos formatos creativos.

Construir conocimiento desde la realidad compleja y las necesidades tal como se vivían y se sentían en primera persona y con los actores afectados permitió construir "una democracia de implicación" (Presno Linera, 2014) y un conocimiento común crítico, más allá los discursos y prácticas neoliberales que defienden el estatus quo y atentan contra los derechos humanos, la sostenibilidad medioambiental promoviendo un modelo de ciudad y de región que excluye a la ciudadanía en su planificación, control y supervisión.

Otro de los factores influyentes para que estas sinergias se dieran quizás fuera la oportunidad de socializar tanto el proceso como los resultados en un evento de 3 días en forma de jornadas abiertas. El fluir de los aprendizajes, los saberes y capacidades tan diversas permitió que, desde conocimientos vivenciales, desde el activismo y el conocimiento técnico, nos introdujéramos en un abordaje integral y en desarrollar una batería de propuestas desde diversas perspectivas. Las fechas de este evento permitió al Grupo Motor calendarizar y programarse. 
La experiencia de este proceso implicó un trabajo bastante laborioso, voluminoso, pero creativo. La sistematización dejó huella de una experiencia pionera y realmente emancipatoria para los participantes del Grupo Motor y de aquellos actores que se involucraron. El hecho de sistematizar estas dinámicas y hacerse oír es el primer eslabón. Aún queda mucho por hacer, ya que consideramos que muchos laboratorios ciudadanos en esta perspectiva debieran emergen y expandirse.

Al mismo tiempo, el proceso de investigación fue atravesado por multitud de preguntas y algunas certezas que emanaron de los ejes de conflicto mencionado más arriba: Especulación, Inmigración, Precariedad, referenciado en tres formatos accesibles a la ciudadanía: un mapa alternativo de la provincia de Málaga, un libro con los testimonios de los participantes y un video con los rostros y voces de los actores involucrados, investigados e investigadores al mismo tiempo. Entre estas pocas certezas, se han evidenciado la estrecha interrelación de los tres ejes de conflictos que operan al margen de las necesidades humanas y sociales y afectan de manera inequívoca y salvaje al territorio y los habitantes de este. No obstante, existe la esperanza de la posibilidad de procesos de autoorganización social, resistencia, intervención y transformación de nuestra realidad ante la mayor intensidad de la explotación, la discriminación y el caos urbanístico determinado por las fuerzas del mercado y las políticas capitalistas posmodernas. En toda la provincia de Málaga se pueden observar resistencias desde los movimientos sociales ante los especuladores urbanísticos cuyos intereses residen en conseguir grandes plusvalías en el menor tiempo posible. De forma difusa e incipiente las comunidades de inmigrantes han madurado sus propias formas de organización y reclamación de sus derechos sociales y políticos y dotar de la población inmigrante de una capacidad de expresión hasta ahora inexistente. Poco a poco, trabajadores precarios comenzaron a crear espacios y tiempos donde se han activado formas incipientes de organización y enunciación, que combinan de manera creativa lo mejor de la tradición sindical y la fuerza de los nuevos movimientos ciudadanos que permitan avanzar juntos en el camino hacia Otra Málaga.

Podríamos pensar a partir de nuestra experiencia investigadora y las evidencias anteriormente descritas, en la necesidad y posibilidad de investigaciones construidas sobre una base de consolidación democrática, como una verdadera escuela de ciudadanía y "democracia de lo común" (Subirats, 2011) donde la cuestión epistemológica sobre la que se apoye defina de primera hora el "para qué" y el "para quién" de dichos espacios. En este sentido, la universidad requiere más que nunca de la confluencia de estas experiencias, que desborden los discursos, abran el abanico y construyan conocimiento científico riguroso tanto teórico, como práctico desde lugares polifónicos. Por lo tanto, más allá de jerarquizar el poder de la palabra, del saber, es imperiosa la necesidad de 
involucrarse en nuevos estilos de desarrollo comunitario que sirvan a delinear políticas y prácticas equitativas, en aras de la justicia social y la democracia real.

Por último, nos gustaría subrayar que el reto a lo que nos enfrentamos los investigadores comprometidos consiste convertir a estas experiencias en espacios de poder vinculante, que influya en las políticas públicas y/o moldeen las agendas políticas locales, otorgando una oportunidad de implicar a la ciudadanía no solo desde el punto de vista consultivo, sino como verdadero motor educativo con poder en la toma de decisiones y en la construcción de territorio que desea vivir.

\section{Bibliografía}

AA.VV. (2009). Metodologías Participativas. Manual. CIMAS-Observatorio Internacional de Ciudadana y Medio Ambiente Sostenible. Disponible en http://www.redcimas.org/wordpress/wp-content/uploads/2012/09/manual_2010.pdf

Alfama i Guillén, E. ( 2009). "Hacia la perspectiva de género en el estudio de los movimientos sociales. La participación de las mujeres en la Plataforma en Defensa de l'Ebre". Revista Española de Investigaciones Sociológicas (Reis), 125: 117-129

Ander-Egg, E. (1990). Repensando la Investigación - Acción - Participativa. Comentarios, críticas y sugerencias. Vitoria-Gasteiz: Servicio de Publicaciones del Gobierno Vasco.

Azcueta, M. (2004). Combate Global contra la Pobreza. Las soluciones existen. La experiencia de Villa El Salvador. San Salvador: Escuela Mayor de Gestión Municipal. UNESCO

Borio, G. (2004). La coinvestigación como acción política. Reviste Derive Aproddi. Roma, Milán. En Nociones comunes. Experiencias y ensayos entre investigación y militancia (pp 67-74). Madrid: Traficantes de sueños.

Canal, R. (2017). «¿Queremos decir lo mismo cuando hablamos de participación? Perspectivas de activistas, técnicos y políticos locales reveladas con metodología Q». Revista Española de Investigaciones Sociológicas, 158: 23-40. http://dx.doi.org/10.5477/cis/reis.158.23

Capra, F. (1992). El punto crucial. Ciencia, sociedad y cultura naciente. Buenos Aires: Ed. Estaciones.

Cohen, L., Manion, L. y Morrison, K. (2000). Research Methods in Education. London: Routledge.

De Sousa Santos, B. (2005). El milenio huérfano. Ensayos para una nueva cultura política. Madrid: Trata/ILSA.

De Sousa Santos, B. (2007). El derecho y la globalización desde abajo. Hacia una legalidad cosmopolita. Barcelona: Ed. Anthropos.

De Sousa Santos, B. (Ed.) (2008). Another knowledge is possible: Beyond Northern epistemologies. London: Verso.

Fals Borda, O. (1990). El problema de cómo investigar la realidad para transformarla por la práxis. Bogotá: Tercer Mundo Editores.

Freire, P. (1973). Pedagogía del oprimido. Buenos Aires: Ed. Siglo XXI. 
Ganuza, E.; Paño, P.; Olivari, L.; Buitrago, L.; Lorenzana, C. (2010). La democracia en acción. Una visión desde las metodologías participativas. Antígona procesos participativos, 2010. Versión Completa En Www.Antigona.Org.Es

Gil Zafra, M. Á. (1998). "Planificación estratégica: el método DAFO". Cuadernos de la Red, 5: 48-59.Madrid: Red CIMAS.

Habegger, S. y Mancila, I. (2005). "La cartografía social como estrategia para diagnosticar nuestro territorio". En Celada, B. \& Habegger, S. (coord.) Interpretando a Freire. Haciendo camino desde la colectividad. Seminario de Paulo Freire. Málaga: Asociación de reflexión e innovación educativa, (pp.29-36). Colectivo Sur Ediciones. Licencia Creative- Commons, ISBN-10: 84-689-5695-3/ ISBN-13: 978-84-689-5695-4.

Habegger, S. (2008). La cartografía del territorio como práctica participativa de resistencia. Procesos en metodologías implicativas, dispositivos visuales y mediación pedagógica para la transformación social. Tesis doctoral Universidad de Málaga disponible en http://riuma.uma.es/xmlui/handle/10630/2555

Hernández, D. (2002). "Estilos y coherencias en las metodologías creativas". En Rodríguez Villasante, T. Metodologías y presupuestos participativos, construyendo ciudadanía $/ 3$ (pp. 17-43) Madrid: Ed. IEPALA-CIMAS Madrid.

Holloway, J. (2002). Cambiar el mundo sin tomar el poder. El significado de la revolución hoy. Madrid: Ed. Viejo Topo.

Ibáñez, J. (1986). "Perspectivas de la investigación social: el diseño en las tres perspectivas”. En García Ferrando, M.; Ibáñez, J. y Alvira, F., (comp.) El análisis de la realidad social Métodos y técnicas de investigación (pp.31-54) Madrid: Ed. Alianza

Kemmis, S. y McTaggart, R. (2005). "Participatory action research: communicative action and the public sphere". In: Denzin, N. K., and Lincoln, Y. S., (eds.) The Sage Handbook of Qualitative Research ( $3^{\text {rd }}$ ed) (pp. 559-603). Thousand Oaks, California, USA: Sage Publications.

Marchioni, M. (1992). La Audición. Un método de investigación participativa y comunitaria. Teoría, metodología y práctica. Canarias. Ed. Benchomo.

Martí, J. (2000). "La Investigación Acción Participativa. Estructuras y fases". En Rodríguez Villasante T, Montañés, M. y Martí, J. (coord.) La investigación social participativa. Construyendo ciudadanía 1 (pp. 73-119). Madrid: Viejo Topo.

Martín Gutiérrez, P. (1998). "Mapas sociales y análisis de redes en la IAP". Cuadernos de la Red, 5: 60-69. Madrid: Red CIMAS.

Martín Gutiérrez, P. (2001). Mapas sociales y ejemplos prácticos en Prácticas locales de creatividad social. Construyendo ciudadanía 2. Madrid: Ed. Viejo Topo.

Maturana, H. R. (1996). El sentido de lo humano. ( $8^{\mathrm{a}}$ ed.). Santiago de Chile: Dolmen ediciones S.A.

Matus, C. (1995). Plan estratégico situacional 95. Guía análisis teórico. Caracas: Ed. Altair.

Max-Neef, M. A. (1986). Desarrollo a escala humana: conceptos, aplicaciones y algunas reflexiones. Uruguay: Ed. Nordan Comunidad.

Pindado, F. (2005). "Gobierno Local y Participación ciudadana". En: MARTí, J. Participación y Desarrollo comunitario en medio urbano. Experiencias y Reflexiones. Construyendo ciudadanía/7. Madrid: Ed. IEPALA/CIMAS.

Presno Linera, M. Á. (2014). Partidos políticos y movimientos ciudadanos en la sociedad del riesgo y la desconfianza. Fundamentos: Cuadernos monográficos de teoría del estado, derecho público e historia constitucional, 8, 213-252 
Rodríguez Villasante, T. (1994). Las ciudades hablan. Caracas: Ed. Nueva Sociedad.

Rodríguez Villasante, T. (1995). Las democracias participativas. De la participación ciudadana a la alternativas de la sociedad. Madrid: Ediciones Hoac.

Rodríguez Villasante, T. (2002). Sujetos en movimiento. Redes y procesos creativos en la complejidad social. Construyendo ciudadanía/4. Montevideo: Ed. Cimas-Nordan.

Rodríguez Villasante, T. (2006). Desbordes creativos. Estilos y estrategias para la transformación social. Madrid: Ed. Catarata.

Rodríguez Villasante, T. (2017). Democracias transformadoras . Experiencias emergentes $y$ alternativas desde los comunes. Barcelona: Viejo Topo.

Romero-Frías, E. \& Robinson-García, N. (2017). "Laboratorios sociales en universidades: Innovación e impacto en Medialab UGR [Social Labs in Universities: Innovation and impact in Medialab UGR]". Comunicar, 51, 29-38. https://doi.org/10.3916/C512017-03

Subirats, J. (2011). Otra sociedad, ¿otra política? De "no nos representan" a la democracia de lo común. Barcelona: Icaria.

\section{Notas biográficas}

SABINA ANDREA HABEGGER LARDOEYT. Doctora en Ciencias de la Educación por la Universidad de Málaga. Es Especialista Universitaria de Posgrado por la Universidad de Pablo Olavide en Investigación Participativa y Desarrollo Local. Viene trabajando desde los últimos 15 años con las metodologías participativas, como asesora, coordinadora y técnica dinamizadora con Administraciones Locales y Nacionales de España y Argentina (Planes Comunitarios, Presupuestos Participativos, Descentralización, Planificación Estratégica en Turismo y Gestión Participativa de Fondos Europeos -EDUSI-).

Actualmente trabaja como técnica en participación ciudadana en el Ayuntamiento de Casabermeja y forma parte activa de Rizoma Fundación.

IULIA MANCILA. Doctora en Ciencias de la Educación por la Universidad de Málaga y Máster: European Counsellor for Multicultural Affairs por The Padagogische Hochschule Linz (Austria) y Universidad de Málaga. (España). Actualmente es profesora en el Departamento de Didáctica y Organización Escolar, Universidad de Málaga (España). Ha participado en varios proyectos de investigación nacionales e internacionales en temáticas relacionadas con la inclusión y justicia social, los enfoques críticos de la educación intercultural, la "segunda generación" de inmigrantes, los jóvenes en riesgo de exclusión, metodologías cualitativas y participativas.

Recibido: 03-01-2018

Aceptado: 18-02-2019 
